\title{
Simple exactly solvable models of non-Fermi-liquids
}

\author{
D. Lidsky and J. Shiraishi \\ Institute for Solid State Physics, University of Tokyo, Roppongi, Minato-ku, Tokyo 106, Japan \\ Y. Hatsugai \\ Department of Applied Physics, University of Tokyo, 7-3-1 Hongo, Bunkyo-ku, Tokyo 113, Japan \\ M. Kohmoto \\ Institute for Solid State Physics, University of Tokyo, Roppongi, Minato-ku, Tokyo 106, Japan
}

(Received 7 May 1997)

\begin{abstract}
We generalize the model of Hatsugai and Kohmoto and find ground states which do not show the properties of Fermi liquids. We work in two space dimensions, but it is straightforward to generalize to higher dimensions. The ground state is highly degenerate and there is no discontinuity in the momentum distribution; i.e., there is no Fermi surface. The Green's function generically has a branch cut. [S0163-1829(98)11303-6]
\end{abstract}

Since the discovery of high- $T_{c}$ superconductivity, strongly correlated electron systems have been intensely studied, both experimentally and theoretically. Much attention has been given to two-dimensional models, because the high- $T_{c}$ cuprates have a layered structure. One of the most studied models is the Hubbard model, but many fundamental questions have not yet been answered. A special version of the model, in the limit that the space dimensionality goes to infinity, has recently been studied. ${ }^{1}$ There are attempts to compare the results with experimental data, in spite of the fact that real materials have at most three dimensions. Similarly, a simple exactly solvable model which describes a metal-insulator transition, and has an altered ground-state momentum distribution, was proposed. ${ }^{2}$ Like the Sherrington-Kirkpatrick model of a spin glass, ${ }^{3}$ the interparticle interaction is independent of distance. The Hamiltonian is

$$
\begin{gathered}
H=\sum_{k} H_{k}, \\
H_{k}=\varepsilon(k)\left(n_{k \uparrow}+n_{k \downarrow}\right)+U n_{k \uparrow} n_{k \downarrow},
\end{gathered}
$$

where $n_{k \sigma}=c_{k \sigma}^{\dagger} c_{k \sigma}$. This gives non-Fermi-liquid behavior in any dimension. Since the Hamiltonian is diagonal in $k$ space, we refer to this model as the HK model. A similar model was discussed by Baskaran ${ }^{4}$ as part of an effort to understand high- $T_{c}$ superconductors. The metal-insulator transition of the HK model which was found in Ref. 2 has been discussed from a scaling point of view by Continentino and Coutinho-Filho, ${ }^{5}$ who also formulated a boson version of the model. Nogueira and $\mathrm{Anda}^{6}$ established the equivalence of the HK model with infinite range hopping to the Hubbard model with infinite range hopping.

In this paper, we extend the $\mathrm{HK}$ model to include coupling between $\mathbf{k}$ modes with the same absolute values of $\mathbf{k}$. Namely, an electron with momentum $\mathbf{k}$ interacts with many other electrons with the same magnitude of momentum. For simplicity, we will work with spinless fermions in two dimensions. Generalization to higher dimensions and to include spin are straightforward.

We study

$$
H=\sum_{\mathbf{k}} \varepsilon(\mathbf{k}) n_{\mathbf{k}}+\frac{1}{2 V} \sum_{\mathbf{k}, \mathbf{k}^{\prime}} f_{\mathbf{k}, \mathbf{k}^{\prime}} n_{\mathbf{k}} n_{\mathbf{k}^{\prime}}
$$

where $V$ is the volume of the system. The summations are taken over $0<|\mathbf{k}|<k_{c}$, where $k_{c}$ represents the short-range cutoff. It is interesting to note that if $n_{\mathbf{k}}$ is replaced by the difference between the interacting and the noninteracting distributions, the interaction energy of Eq. (2) has a resemblance to that in the Fermi-liquid theory. In fact, Khodel and Shaginyan, ${ }^{7}$ starting from the Fermi-liquid equation, proposed the existence of "fermion condensation" which gives large degeneracy at the Fermi surface. Within the HartreeFock treatment, Nozieres ${ }^{8}$ criticized such phenomenon. (See also Ref. 9.) The highly degenerate ground state of our model looks similar to the "fermion condensation." However, in our case there is no definite Fermi surface and the model is exactly solvable, so Nozieres' critique does not apply.

In the thermodynamic limit $V \rightarrow \infty$, the interaction term in Eq. (2) can be written

$$
f\left(\mathbf{k}, \mathbf{k}^{\prime}\right)=(2 \pi)^{2} U(k) \delta\left(k-k^{\prime}\right) \frac{g(\phi)}{k},
$$

where $k=|\mathbf{k}|, k^{\prime}=\left|\mathbf{k}^{\prime}\right|$, and $\phi$ is the angle between $\mathbf{k}$ and $\mathbf{k}^{\prime}$. The factor $1 / k$ comes from the measure in two-dimensional polar coordinates. The function $g(\phi)$ is periodic $g(\phi)$ $=g(\phi+2 \pi)$ and satisfies $g(\phi)=g(-\phi)$, assuming parity invariance. Throughout this article we will work with the condition that $g(\phi)$ takes the maximum for $\phi=\pi\left(\mathbf{k}^{\prime}=\right.$ $-\mathbf{k}$ ), and monotonically decreases toward $\phi=0$. For this class of models, we can obtain the exact ground states with high degeneracy. The momentum distribution is shown to be continuous and does not have a jump in contrast to the Fermi liquids. It will be shown that the Green's functions generically have a branch cut instead of a pole.

Let us obtain the ground state and its momentum distribution. Due to the angular dependence of the interaction $g(\phi)$ the electrons will bunch up in an arc of angle $\phi_{k}$. So, the momentum distribution is 


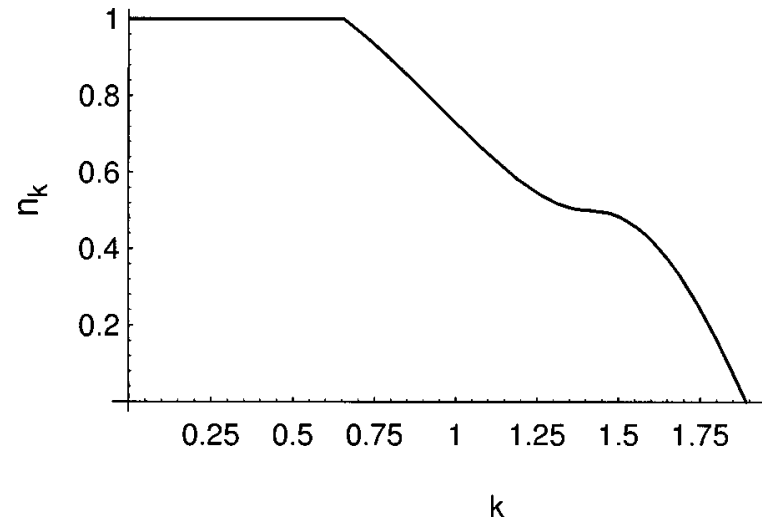

FIG. 1. Ground-state momentum distribution for $g(\phi)=\left(\pi^{2}\right.$ $\left.-\phi^{2}\right)^{-1 / 2}$ and $U=1 / 2$.

$$
n_{k}=\frac{\phi_{k}}{2 \pi} \text {. }
$$

The ground-state energy including a chemical potential $\mu$ is

$$
\begin{aligned}
\langle H-\mu N\rangle_{G}= & \frac{V}{(2 \pi)^{2}} \int_{0}^{k_{c}} d k k\left[(\varepsilon(k)-\mu) \phi_{k}\right. \\
& \left.+U(k) \int_{0}^{\phi_{k}} d \phi \int_{0}^{\phi} d \phi^{\prime} g\left(\phi^{\prime}\right)\right]
\end{aligned}
$$

where \langle\rangle$_{G}$ is the ground-state expectation value, and $\varepsilon(\mathbf{k})$ is assumed to be independent of $\phi$. Since $\phi_{k}$ must minimize Eq. (5), we obtain, by taking the derivative of $\langle H-\mu N\rangle_{G}$ with respect to $\phi_{k}$,

$$
\varepsilon(k)+U(k) \int_{0}^{\phi_{k}} g(\phi) d \phi=\mu .
$$

The ground state is specified by $\phi_{k}$, but it is highly degenerate due to the freedom of choice of an arc of angle $\phi_{k}$ at each $k$. The momentum distribution (4) is obtained by solving Eq. (6). Let $k_{1}$ be the largest value of $k$ which gives $\phi_{k}=2 \pi$ and let $k_{0}$ be the smallest value of $k$ which gives $\phi_{k}=0$. Then $n_{k}=1$ for $k<k_{1}, 0<n_{k}<1$ for $k_{1}<k<k_{0}$, and $n_{k}=0$ for $k_{0}<k$. Thus $n_{k}$ has cusps at $k_{1}$ and $k_{0}$. We also

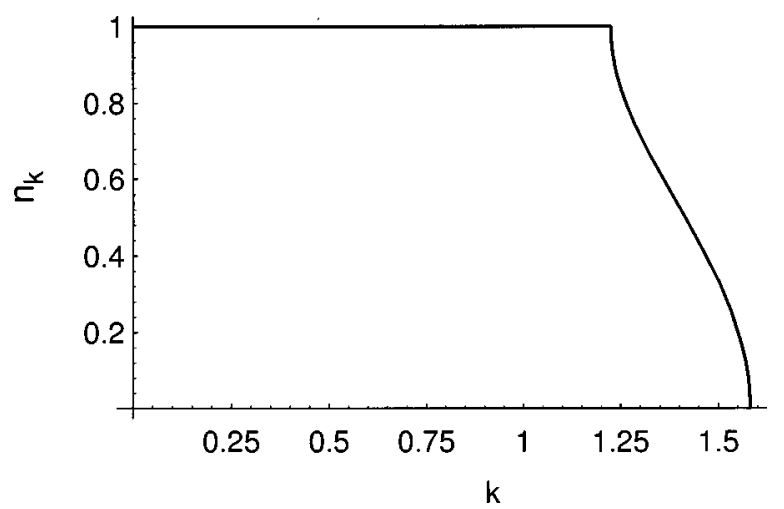

FIG. 2. Ground-state momentum distribution for $g(\phi)$ $=\sin (\phi / 2)$ and $U=1 / 8$.

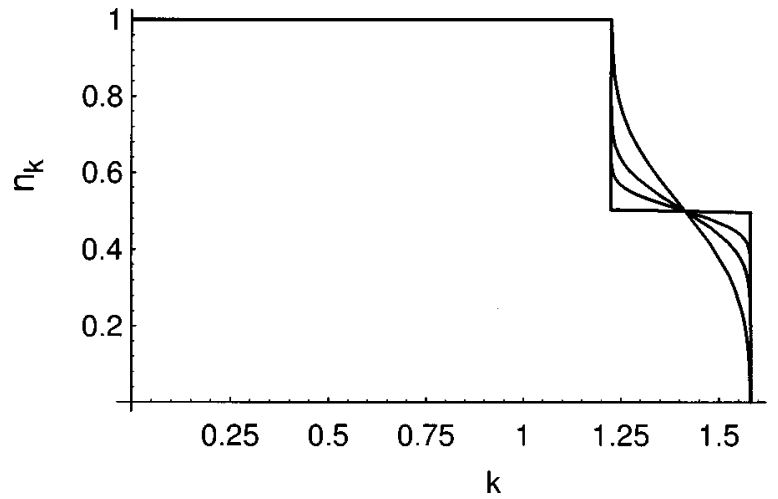

FIG. 3. Ground-state momentum distribution for $g(\phi)$ $=\left(1 / \sigma \pi^{1 / 2}\right) \exp \left[(\pi-\phi)^{2} / \sigma^{2}\right]$ and $U=1 / 2, \sigma=\pi / 512, \pi / 8, \pi / 4$, $\pi / 2$.

define $k_{1 / 2}$ by the condition $\phi_{k_{1 / 2}}=\pi$. It is possible to have $n_{k}<1$ for all $k$, i.e., the absence of $k_{1}$, if the interaction is strong enough.

Suppose that $U(k)$ is not a singular function and $g(\phi)$ is a function whose singularity is weaker than the integrable ones. Then $\phi_{k}$, the solution of Eq. (6), cannot have a discontinuity. Namely, $n_{k}=\phi_{k} / 2 \pi$ is continuous and we do not have a Fermi surface associated with discontinuity of momentum distributions.

Let us observe whether the Mott metal-insulator transition may occur or not. Note that due to the cutoff $k_{c}$, there is a bandwidth $W=\varepsilon\left(k_{c}\right)-\varepsilon(0)$. Let us examine the following two cases:

(i) If the interaction satisfies the conditions $f_{\mathbf{k}, \mathbf{k}^{\prime}} \sim O(1)$ for $\mathbf{k} \neq-\mathbf{k}^{\prime}$ and $f_{\mathbf{k},-\mathbf{k}}>V W$, the angle $\phi_{k}$ is exactly equal to $\pi$ for any $k$ at the half filling. A finite amount of energy is needed to add one particle to this ground state. We should note that the condition $f_{\mathbf{k},-\mathbf{k}}>V W$, namely that $f$ has a divergence of order $V$ at $\mathbf{k}=-\mathbf{k}^{\prime}$, means that the function $g(\phi)$ contains a $\delta$-function-like singularity at $\phi=\pi$.

(ii) If the condition $f_{\mathbf{k},-\mathbf{k}}>V W$ is not satisfied for some $k$, it costs almost no energy to add a particle to the ground state. Namely, there is no gap between the ground state and the low-energy states. Thus, the Mott transition will not happen.

To show the smooth falling off of the momentum distribution of the ground state, we will give some examples. For simplicity, $U(k)$ is taken to be constant $U$.

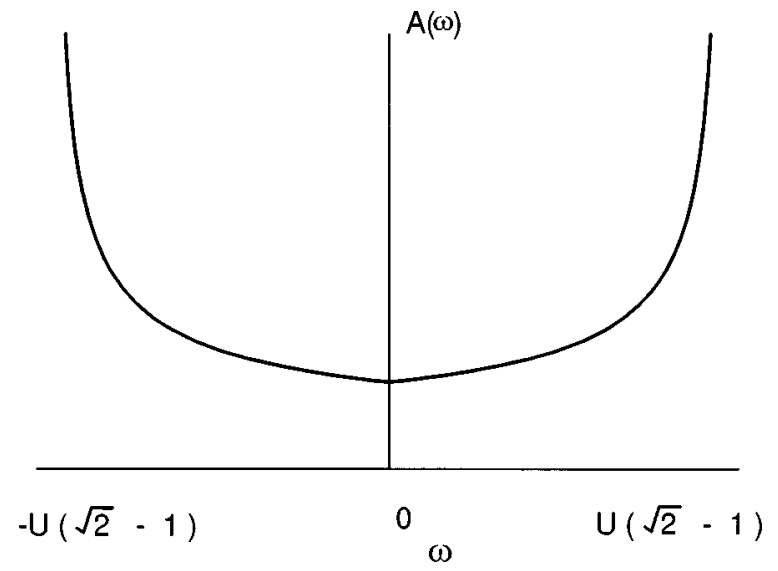

FIG. 4. Spectral function for $g(\phi)=\sin (\phi / 2)$, at $k_{1 / 2}$. 


$$
\text { (I) } \begin{aligned}
g(\phi)= & \frac{1}{\left(\pi^{2}-\phi^{2}\right)^{1 / 2}} \text { for } 0<\phi<\pi, \\
= & \frac{1}{\left(\pi^{2}-(2 \pi-\phi)^{2}\right)^{1 / 2}} \\
& \text { for } \pi<\phi<2 \pi .
\end{aligned}
$$

This interaction has an integrable singularity at $\phi=\pi$, causing the tangent to $n_{k}$ to be horizontal at $n_{k}=1 / 2$. The derivative of $n_{k}$ is discontinuous at $k_{1}$ and $k_{0}$. See Fig. 1 .

$$
\begin{aligned}
& n_{k}= \begin{cases}1-\frac{1}{2} \sin \frac{\mu-\varepsilon(k)}{U} & \text { for } k_{1}<k<k_{1 / 2}, \\
\frac{1}{2} \sin \frac{\mu-\varepsilon(k)}{U} & \text { for } k_{1 / 2}<k<k_{0} .\end{cases} \\
& \text { (II) } g(\phi)=\sin \frac{\phi}{2} \text {. }
\end{aligned}
$$

The derivative of $n_{k}$ is discontinuous at $k_{1}$ and $k_{0}$. We have $\lim _{k \rightarrow k_{1}^{+}} d n / d k \propto-\left(k-k_{1}\right)^{-1 / 2}$ and $\lim _{k \rightarrow k_{0}^{-}} d n / d k \propto-\left(k_{0}\right.$ $-k)^{-1 / 2}$. At $k=k_{1 / 2}$ the second derivative changes sign. See Fig. 2.

$$
n_{k}= \begin{cases}1-\frac{1}{\pi} \arccos \left(\frac{\mu-\varepsilon(k)}{2 U}-1\right) & \text { for } k_{1}<k<k_{1 / 2}, \\ \frac{1}{\pi} \arccos \left(1-\frac{\mu-\varepsilon(k)}{2 U}\right) & \text { for } k_{1 / 2}<k<k_{0} .\end{cases}
$$

$$
\text { (III) } g(\phi)=\frac{1}{\sigma \pi^{1 / 2}} \exp \left[-\frac{(\pi-\phi)^{2}}{\sigma^{2}}\right] \text {. }
$$

In this example, we set $\varepsilon(k)=k^{2} / 2 m$. As $\sigma \rightarrow 0$, the interaction approaches a $\delta$ function and the Hamiltonian approaches the original HK model. This can be seen in Fig. 3. For the smallest $\sigma$, the momentum distribution appears to have two pseudo-Fermi surfaces, as in the HK model. Note that, if $\sigma$ is finite, there is no Mott transition at half filling, as mentioned earlier. We may obtain $\phi_{k}$ by inverting

$$
k= \begin{cases}\sqrt{2 m\left[\mu-\frac{U}{2} \operatorname{erf}\left(\frac{\pi-\phi_{k}}{\sigma}, \frac{\pi}{\sigma}\right)\right]} & \text { for } \phi_{k}<\pi, \\ \sqrt{2 m\left[\mu-\frac{U}{2} \operatorname{erf}\left(0, \frac{\pi}{\sigma}\right)-\frac{U}{2} \operatorname{erf}\left(0, \frac{\phi_{k}-\pi}{\sigma}\right)\right]} & \text { for } \phi_{k}>\pi,\end{cases}
$$

where $\operatorname{erf}(x, y)=2 \pi^{-1 / 2} \int_{x}^{y} e^{-t^{2}} d t$.

Next, let us study the Green's function. The ground states of our models are highly degenerate. For each $k$ in the partially occupied zone, we may rotate the electrons in $k$ space by an arbitrary angle and obtain another ground state. We will show that after averaging over all such ground states, the single-particle Green's function develops a branch cut, a signature of a non-Fermi-liquid.

The Green's function is

$$
G(\mathbf{k}, t)=\theta(t) G^{>}(\mathbf{k}, t)-\theta(-t) G^{<}(\mathbf{k}, t),
$$

where $G^{\gtrless}(\mathbf{k}, t)$ are the correlation functions

$$
\begin{aligned}
& G^{>}(\mathbf{k}, t)=\int_{0}^{2 \pi} \frac{d \varphi}{2 \pi}\left\langle\varphi\left|e^{i(H-\mu N) t} c_{\mathbf{k}} e^{-i(H-\mu N) t} c_{\mathbf{k}}^{\dagger}\right| \varphi\right\rangle, \\
& G^{<}(\mathbf{k}, t)=\int_{0}^{2 \pi} \frac{d \varphi}{2 \pi}\left\langle\varphi\left|c_{\mathbf{k}}^{\dagger} e^{i(H-\mu N) t} c_{\mathbf{k}} e^{-i(H-\mu N) t}\right| \varphi\right\rangle .
\end{aligned}
$$

Suppose that the electrons are on an $\operatorname{arc}$ between $\varphi$ and $\varphi$ $+\phi_{k}$ of a circle with radius $k$. Using circular symmetry, the correlation functions are obtained and their Fourier transforms $G^{\gtrless}(\mathbf{k}, \omega)=\int_{-\infty}^{\infty} d t e^{i \omega t} G^{\gtrless}(\mathbf{k}, t)$ are

$G^{>}(\mathbf{k}, \omega)=-2 \operatorname{Im} \int_{0}^{2 \pi-\phi_{k} d \varphi} \frac{1}{2 \pi} \frac{1}{\omega-\varepsilon(k)+\mu-U P_{k}(\varphi)+i \delta}$,

$$
G^{<}(\mathbf{k}, \omega)=-2 \operatorname{Im} \int_{2 \pi-\phi_{k}}^{2 \pi} \frac{d \varphi}{2 \pi} \frac{1}{\omega-\varepsilon(k)+\mu-U P_{k}(\varphi)+i \delta}
$$

where

$$
P_{k}(\varphi)=\frac{1}{2} \int_{\varphi}^{\varphi+\phi_{k}} d \varphi^{\prime} g\left(\varphi^{\prime}\right)
$$


The integrands in Eq. (16) have a pole at $\omega=\varepsilon(k)-\mu$ $+U P_{k}(\varphi)$. Thus, after integration over $\varphi$ we have a branchcut in the Green's function instead of a pole which corresponds to a quasiparticle.

The spectral function, relevant to photoemission, is

$$
\begin{aligned}
A(\mathbf{k}, \omega) & =G^{>}(\mathbf{k}, \omega)+G^{<}(\mathbf{k}, \omega) \\
& =-2 \operatorname{Im} \int_{0}^{2 \pi} \frac{d \varphi}{2 \pi} \frac{1}{\omega-\varepsilon(k)+\mu-U P_{k}(\varphi)+i \delta} .
\end{aligned}
$$

For example, if one takes the interaction $g(\theta)=\sin (\theta / 2)$,

$$
\begin{aligned}
& \left.A(\mathbf{k}, \omega)\right|_{\mathbf{k}=\mathbf{k}_{1 / 2}} \\
& \quad= \begin{cases}\frac{\sqrt{2}}{U \sqrt{1-\frac{1}{2}(1+|\omega| / U)^{2}}} & \text { if }|\omega|<U(\sqrt{2}-1), \\
0 & \text { otherwise. }\end{cases}
\end{aligned}
$$

This is shown in Fig. 4.

This work was supported by a Grant-in-Aid from the Ministry of Education, Science and Culture of Japan. D. L. was supported by the Japan Society for the Promotion of Science.
${ }^{1}$ For a review see, e.g., A. Georges, G. Kotliar, W. Krauth, and M. Rozenberg, Rev. Mod. Phys. 68, 13 (1996).

${ }^{2}$ Y. Hatsugai and M. Kohmoto, J. Phys. Soc. Jpn. 61, 2056 (1992).

${ }^{3}$ D. Sherrington and S. Kirkpatrick, Phys. Rev. Lett. 35, 1792 (1975).

${ }^{4}$ G. Baskaran, Mod. Phys. Lett. B 5, 643 (1991); V. N. Muthukumar and G. Baskaran, Mod. Phys. Lett. B 8, 699 (1994). Baskaran's Hamiltonian,

$$
\begin{gathered}
H=\sum_{k}(\varepsilon(k)-\mu) c_{k \sigma}^{\dagger} c_{k \sigma}+J\left(\sum_{k} S_{k}\right)^{2}-J \sum_{k} S_{k}^{2} \\
S_{k} \equiv c_{k \alpha}^{\dagger} \sigma_{\alpha \beta} c_{k \beta},
\end{gathered}
$$

would be the same as the model of Hatsugai and Kohmoto were it not for the second term which partially lifts the degeneracy.

${ }^{5}$ M. A. Continentino and M. D. Coutinho-Filho, Solid State Commun. 90, 619 (1994).

${ }^{6}$ F. S. Nogueira and E. V. Anda, Int. J. Mod. Phys. B 10, 3705 (1996).

${ }^{7}$ V. A. Khodel and V. R. Shaginyan, JETP Lett. 51, 553 (1990).

${ }^{8}$ P. Nozieres, J. Phys. (Paris), Colloq. 2, 443 (1992).

${ }^{9}$ D. V. Khveshchenko, R. Hlubina, and T. M. Rice, Phys. Rev. B 48, 10766 (1993). 